\title{
The Effects of an Ultrasound and Radient Heat Therapy on Knee Osteoarthritis in Female
}

\author{
Anita Bai ${ }^{1}$, Jetindar Puri ${ }^{2}$, Kailash Kumar ${ }^{3}$, Sumaiya Afzal $^{4}$, Reeta ${ }^{5}$, \\ Dr Zuhaira Faruqui ${ }^{6}$, Pardeep Kumar ${ }^{7}$, Wakash Lal ${ }^{8}$ \\ ${ }^{1}$ senior physiotherapist at jinnah postgraduate medical center Karachi \\ ${ }^{2}$ Physiotherapist at jinnah postgraduate medical center Karachi \\ ${ }^{3}$ Physiotherapist at jinnah postgraduate medical center Karachi \\ ${ }^{4}$ Physiotherapist at jinnah postgraduate medical center Karachi \\ ${ }^{5}$ senior lecturer at IPMR Dow University health and sciences Karachi \\ ${ }^{6}$ Physiotherapist at ziauddin hospital clifton Karachi \\ ${ }^{7}$ Physiotherapist at Dubai hospital UAE \\ ${ }^{8}$ senior lecturer at IPMR Dow University health and sciences Karachi
}

\begin{abstract}
Objective; To Determine the Effectiveness of Ultrasound and Radiant Heat Therapy On Osteoarthritis In Female Patients.

DESIGN Study Design Was Experimental Based. Randomized Control Trial.

Setting; This study was done in Jinnah post graduate medical center (JPMC) and institute of physical medical rehabilitation.

Sample Size; sample size was 60 subjects.

Duration of Study; The duration of study was 6 months

Subjects and Participants; Only female participants were selected ranged in age of 40 to 60 years old, weight was less than 90 and female with only chronic condition were taken.

Result; This study was an experimental design.60 participants completed the trial.60 subjects were divided into two groups experimental and control group. Experimental group who was received intervention ultrasound therapy with strengthening exercise showed great difference in decreasing pain increasing activities then the control group who was received intervention Radiant heat only. The statically analyze done with Mann-Whitney test which showed great improvement. The level of Significance 0.02

Conclusion

This study strongly suggest that ultrasound treatment was more effective then Radiant heat treatment. As result were significant so it suggests ultrasound treatment improves knee function and decrease pain significantly in patients with knee osteoarthritis
\end{abstract}

\section{Introduction}

Osteoarthritis is the most common rheumatic disease.1 The condition is associated with pain and inflammation of joint capsule ${ }^{[2]}$ Impaired muscular stability ${ }^{3,4}$. Decrease range of motion ${ }^{[5]}$ Among older people, this chronic disease of the musculoskeletal system is one of the most frequent causes of dependency ${ }^{[6]}$

In 1994, Ettinger and afable proposed a model to explain the process by which osteoarthritis of the knee may lead to decrease to functional activities, this model focus on pain which limits use of lower extremities which affects three main components that is aerobic capacity, muscle strength, and flexibility ${ }^{\text {[7-10] }}$

This model postulates that reduced activity maximum impact on daily living activities such as going up and down targeting up from chair standing and walking, from last few years' therapeutic exercise became beneficial treatment for osteoarthritis of knee ${ }^{[11]}$

Numerous studies have investigated the effect of different type of exercise in knee osteoarthritis, the program consist of aerobic,resistance,flexibility exercise ${ }^{[12]}$ The weakness of Quadriceps Muscle is well noticed in patients of knee osteoarthritis ${ }^{[13,14,15]}$ Quadriceps muscle is strongly associated with pain and important determinant of disability ${ }^{[16]}$

European league Againts rheumatism (EULAR) recommendation state that both pharmacological and non-pharmacological are needed for treatment of osteoarthritis ${ }^{[17]}$ Non pharmacological intervention includes acupunture,low level lesser therapy,transcutanous electrical nerve stimulation and ultrasound ${ }^{[18]}$ Non-steroidal anti-inflammatory drugs most common use of knee osteoarthritis may have deleterious effect on cartilage metabolism ${ }^{[19,20]}$

The knee joint is designed for mobility and stability its function is lengthens and shortens the lower extremity to raise and lower the body to move the foot in space 
Along with hip and ankle it supports the body when standing and it is primary functional unit in walking climbing running and sitting activities

The bones of the knee joint consist of distal femur with its two condyles the proximal tibia with its two tibial plateaus and large sesamoid bone in the quadriceps tendon,patella.

The knee joint is biaxial joint,modified hinge joint with two interposed menisci supported by the ligaments and muscles.

Anteroposterior stability is provided by the cruciate ligaments, mediolateral stability is provided by medial lateral collateral ligaments

The convex bony partner is composed of two asymmetrical condyles on the distal end of femur the medial condyle is larger than the lateral condyle which contributes locking machanism of knee joint

The concave bony partner is composed of two tibial plateaus on the proximal tibia with their respective fibrocartilagious menisci the medial plateau is larger than lateral plateau Menissci improve the congruency of the articulating surfaces The rotation that occurs between the femoral condyles and tibia during final degree of extension is called the locking,scew home machanism The patella is a sesamoid bone in the quadriceps tendon,it is embedded in the anterior portion of joint capsule connected to the tibia by the ligamentum patella many bursae surround the patella The aligment of the patella in the frontal plane is influenced by line of pull of quadriceps muscle group by its attachment to the tibial tubercle by the patellar tendon,the result of these two forces is a browstring effect on patella cousing it to track laterally The Q Angle is formed by two intersecting lines one is from anterior superior iliac spine to the mid patella other is from tibial tubercle through the mid patella The normal Q Angle which tends to be greater in women then the men Structurally an increased Q Angle occurs with wide pelvis,femoral anteversion,coxa vara,genu valgum,lateraly displaced tibial tuberosity,pronated subtalar joint. Knee joint center moves medialy relative to the foot during weight bearings activities also increases Q Angle A weakness of hip abductors and external rotators may result in adduction of femur and valgus at the knee contribute to increased medial rotation The quadriceps femoris muscle group is the only muscle crossing anterior to the knee it is prime movers for knee extention, hamstrings and the soleus muscle control knee extention by pulling tibia posteriorly The hamstings muscles are primary knee flexors also influences the rotation of tibia on the femur hamstring is two joint muscle they contract more efficiently during hip flexion and knee flexion ${ }^{[21]}$ In a study done by Maohsiung et al (2005) on 120 subjects.where they determined use of ultrasound to increase effectiveness of isokinetic muscle stregthening exercise for the treatment of osteoarthritis where they formed four groups. 1 received isokinetic muscular strenthening exercise group 2 received isokinetic exercise with continous ultrasound group 3 received isokinetic exercise with pulsed ultrasound and group 4 was control group. They were concluded that changes in ambulation speed in addition changes in ROM and decrease pain group 2,3 had significant improvement and increase effectiveness of isokinetic exercise for functional improvement of knee pulsed ultasound has greater effect then continuous ultrasound ${ }^{[22]}$

In a study done by Gail D et al (2000) In a study done by Judith Falconer PHD (2005) determined effect of ultrasound on mobility in osteoarthritis of knee Randomised control Trail 35 subjects were selected they determine ultrasound increases soft tissue extensibility may be effective adjunct in treatment of knee contacture secondry to connective tissue extensibility ${ }^{[23]}$

\section{Objective}

- To determine the effectiveness of ultrasound with strenthening exercise in comparison with radiant in pain managment

- To determine the effect on ADL activities

- To determine the effect on healthy life style

\section{Hypothesis}

\section{Experimental Hypothesis}

Ultrasound along with strenthening exercise decrease more pain increase functional activities in osteoarthritis as compared to radient heat with strenthening exercise

\section{Null Hypothesis}

Ultrasound along with strenthening exercises not decrease pain and increases functional activities in knee osteoarthritis as compared to radiant heat with strethening exercise.

\section{Oprational Definations}

\section{Oseoarthritis of knee joint.}

Osteoarthritis is maked by two localized, pathological features, the progressive destruction of articular cartilage of bone at the margins of knee joint. The disease process of OA confines itself to the affected joint. However, the impaiement, functional, limitation, and disability related to OA can reach for beyond the perimeter of articular cartilage and subchondral bone ${ }^{[24]}$ 


\section{Therapeutic Ultrasound}

Ultrasound therapy intervention delivered mechanical vibration using an ultrasound device at frequencies between 1.0-3.0 MHz

Crieria for optimal dose: intensity $0.1-3 \mathrm{~W} / \mathrm{cm} 2$, continuous or pulsed output, treatment time between 3-20 minutes and doses between 18-540 joules per session. these doses are based on those commonly reported in clinical literature as optimal dose range has to yet to be established ${ }^{[25,26]}$

\section{Visual Analogue Scale:}

VAS is a chart with the scale marked $0-10$ on it. Patients mark on it considering the intensity of pain, ' 0 ' representing 'no pain' and ' 10 's 'maximum pain. The pain rating was done by the patient on the basis of the pain felt by him or her before and after the treatment sessions ${ }^{[27]}$

\section{Study design:}

Randomized Control Trial

Source of Data:

Study was conducted in Physiotherapy OPD in Jinnah post graduate medical center(JPMC) and Institute of Physical Medicine and Rehabilitation of DOW (IPMR)

\section{Duration of study:}

six months

\section{Sampling Technique:}

Simple random sampling.

Sampling Methods:

Subjects was randomly allocated into two groups

Experimental group U/S with Straitening Exercise

Control group RADIENT Heat with Strengthening Exercise

Sample size

A sample of 60 patients was inducted in the study

\section{Inclusion criteria}

- Only female patients with age $>40$ up to 60

- Unilateral and Bilateral (Both) knees osteoarthritis was taken

- Only chronic stage

- The Patients weight should be less then $<90$

Exclusion Criteria

- All male patients

- Acute stage

- Below age of 40

- $\quad$ Weight $>90$

\section{Data collection Tool}

Patients Related Knee osteoarthritis = Assessment Form

Visual Analog Scale =Evolution of Pain

\section{Data Collection Procedure}

An assessment form was used for data collection. The purpose of this study was explained and written informed consent was obtained from all the participants. The Subjects were assessed before any intervention by seeing, muscle spasm, loss of movement joint stiffness ADL activities before and after treatment. The (VAS) and (ADL) activates was used to assess the subjects to compare results.

\section{Data Analysis}

Data was analyzed by applying Mann-Whitney U test will be used in ordinal scale to see pain. Different subjects study design test was used to analyze data in order to compare the change of out come before and after applying the intervention on both groups; Experimental group on which ultrasound and strengthening exercise was applied and on control group Radiant and strengthening was applied. Data showed significant improvement in pain after two weeks programe conducted on female patients only. Mann-Whitney U Test Showed significant result 0.02 that was checked on Microsoft Excel 2007.Thus we accept experimental hypothesis and reject null hypothesis. 


\section{Result}

The study was an experimental design, to analyze the result from the experiments, Mann-whitely test was used. Mann - Whitney U test simply campers the results from each group to see if they differ significantly. MannWhitney ' $U$ ' test shows probability $\mathrm{p}$ both groups. One was the experimental group who received treatment intervention of ultrasound with stretching exercise other one was the control group who received treatment intervention of radiant heat, when compared both of those intervention the experimental group showed 0.02 level of significant. when calculating this test end up with the numerical value, 'U' which we look up in the probability tables associated with the Mann- Whitney tests this level of significance is also was cheked Microsoft excel 2007. thus we accept the experimental hypothesis which states that ultrasound is batter then radiant heat in knee OA of elderly patient.

\section{Discussion}

After one month of intervention significantly greater improvement was observed in the experimental then the control group for the following variables; arthritic pain, walking and low back flexibility. in patients with the chronic knee pain and weakness of quadriceps result in progressive shortening of periarticular and connective tissue and flexion contracture of the arthritic knee. It may become fibrotic, contracted, and shortened when subjected to immobilization or inactivity due to arthritic join pain resulting in joint capsule contracture and limited ROM, slow sustained stretching was perfumed to treat arthritis knees slower sustained stretching was performed, Golgi tendon organs, which lie in series with extrafusal muscle fibers, resulting in muscle relaxation. its seemed that ultrasound therapy along with the stretching exercises exhibits statistically significant effects, ultrasound therapy which delivered mechanical vibration using ultrasound device at frequencies between $1.0-3 \mathrm{MHz}$. this studies supported the hypothesis that ultrasound Therapy along with stretching exercise were more effective then radiant heat on pain in knee osteoarthritis. Subjects were assessed before and after physical therapy intervention which showed a significant difference in decreasing pain and increasing the activities. there was significant difference was found in the pain on visual analogue scale. 60 subjects were selected for the study which was divided into two equal groups was assessed visual analog scale. After the treatment for the one month the patients were re assessed and data was analyzed by using Mann - Whitney U test which showed 0.02 level of significance

\section{Synopsis of Result:}

Result from Mann Whitney test was performed which is confirmed by Microsoft excess 2007. The data was found to be significant at the level of $p<0.01$ so this test showed level of significant 0.02 . This shows that ultrasound is more effective than radiant heat in treating the patient with osteoarthritis because there was significant difference noted before and after the value of the patients with ultrasound.

\section{Conclusion}

The result of study strongly suggest that Ultrasound therapy with exercise is more effective then Radient heat intervantion.As result is significant so it suggest that ultrasound therapy treatment improves knee function and decreases pain.

\section{Exercise}

During each appointment objects underwent an exercise programme. The performing isometric exercise of the quadriceps muscles. Quadriceps-muscle strengthening is a common goal in the management of osteoarthritis, preventing disuse atrophy and protecting against further progression of degenerative processes ${ }^{[28]}$

Quadriceps-strengthening exercise were performed using metallic interchangeable weights fastened to the ankle of the effected leg. the delorme and watkins principle of 10-repetitive maximum (10RM) was used to determine and standardize the weight used by each subject ${ }^{[29]}$ subjects sat on the edge of a plinth with the back fully supported and the two hands holding the edges of the plinth. Subjects performed isometric knee extebsion exercises from a starting position of $0^{\circ}$ knee flexion. the subject was helped by an investigator lift the weight from $90^{\circ}$ to $180^{\circ}$ extensions. the extension was sustained for 10 seconds (isometric) before the leg was lowered (with help, if required) and rested for 5 seconds. subjects the exercise 10 times to make one bout. A total of 10 bouts were performed with 2 minutes' rest in between bouts (total of 100 contraction ${ }^{\text {) }}{ }^{30]}$.

\section{References}

[1]. Scott jc, Hochberg MC. Arthritis and musculoskeletal disorders.In :Brownson RC,Remington PL,Davis JR,editors.Chronic disease epidermology and control.Washington,DC: American Public Health Association 1993.p.285-305.

[2]. Naredo E, Cabero F,Palop Mj Collado P Cruz ACrespo M:Ultrasonographic findings in knee osteoarthritis; a comparative study with cilinical and radiographic assessment.Osteoarthritis cartilage 2005,13(7):568-574.

[3]. Brandt KD,Heilman DK Slemenda C,katz BP Mazzuca S,Braunstein EM,Byrd D ;A Comparison of lower extremity muscle strength, obesity, and depression scores in elderly subjects with knee pain with and without radiographic evidence of knee osteoarthritis. JRheumatol 2000,;27(8)1937-1946.

[4]. Cowan SM Bennell KL Hodges PW,Crosseley KM McConnell J : Delayed onset of electromyographic activity of vastus medialis 
obliquuus relative to vastus lateralis in subjects with patellofemoral pain syndrome. Arch Phys Med Rehabil 2001,82(2):183-189.

[5]. Steultjens MP,Dekker J,van Baar ME Oostendrop RA,Bijlsma JW: Range of joint motion and disability in patients with osteoarthritis of the knee or hip. Rheumatology (Oxford) 2000.39(9):955-961.

[6]. Ettinger WH,Afable RF Physical Disability from knee osteoarthritis:the role of exercise as an intervention.Med Sci Sports Exerc1994;26;1435-40.

[7]. Beals CA ,Lampman RM,Banwell BF,Braunstein Em Albers JW,Castor CW,Measurment of exercise of tolerance in patients with rheumatoid arthritis or osteoarthritis.J Rheumatol 1985;12:458-61.

[8]. Minor MA Hevitt JE Webel RR Dreisinger TE;Kay DR, Exercise Tolerance and disease related measure in patients with rheumatoid arthritis and osteoarthritis J Rheumatol 1988;15;905-11.

[9]. Messier SP Loeser RF,Hoover JL,Semble EL,Wise CM.Osteoarthritis of the knee; effects on diet,strength,Flexibility.Arch Phys Med Rehabil 1992;73;29-36.

[10]. Phibin EF,Groff GD,Ries MD Miller TE.Cardiovascular fitness and health in patients with end-stage osteoarthritis.Arthritis Rheum 1995;38;799-805.

[11]. Afable RF Benefits of exercise for older patients with osteoarthritis of the knee Clin Geriatr 1993;1;34-40

[12]. Hoffman DF Arthritis and exercise.Prim Care1993;20;895-910

[13]. HurScott DL Rees J,Newham DJ.Sensorimotor changes and functional performance in patients with knee osteoarthritis.Annals of Rheumatic dieases 1997;56:641-648.

[14]. Lewek MD,Rudolph KS,Snyder-Mackler L.Control of frontal plane knee laxity durind gait in patients with medial compartment knee osteoarthritis.Osteoarthritis Cartilage 2004;12:745-751.[pubMed:15325641].

[15]. Selmenda C,Brandt KD,Heilman DK,Mazzuca S,Braunstein EM,et al.Quadriceps weakness and osteoarthritis of the knee.Annals of Internal Medicine 1997;127;97-104[Pubmed:9230035].

[16]. O Reilly SC,Jones A Muir KR,Doherty M Quardriceps weakness in knee osteoarthritis:the effect on pain and disability. Annals of Rhumatic Diseases1998;57(10):588-594[Pub Med:[9893569]

[17]. Jordan KM,Arden NK,Doherty M Bannwarth B,Bijlsma Jw,dieppe p Gunther k Hauselmann H Herrero-Beaumont G Kaklamanis p Lohmander s Leeb B Lequesne M Mazieres B Verbruggen G Zimmerman- Gorska I Dougados M :EULAR Recommendations 2003: an evidence based approach to the managment of knee osteoarthritis:Report of the task force of the standing Committee for the international clinical studies including therapeutic trials (ESCISIT) Ann Rheum Dis 2003,62(12) 1145-1155

[18]. Bjordal JM,Johnson MI Ljunggreen AE Transcutaneous electrical nerve stimulation(TENS) can reduce postoperative analgesic consumption. A meta-analysis with assessment of optimal treatment parameters for the postoperative pain. Eur $\mathrm{J}$ Pain 2003,7(2);181-188.

[19]. Brandt KD Effects of non steroidal anti-inflammatory durugs on chondrocyte metabolism in vitro and vivo. Am J Med 1987;83:29-34

[20]. Bradley JD, Brandt KD,Katz BP,et al Comparison of an antiinflammatory dose of ibuprofen an analgesic dose of ibuprofen and acetaminophen in the treatment of patients with osteoarthritis of knee.N Engl Med 1991;325:87-91.

[21]. Carolyn kisner (2003)Therapeutic exercise Foundation and technique,fourth edition published by jitendar PVI Jaypea, Brothers medical publisher EMCA House New Delhi India

[22]. Mohsiung huang et al (2005) determine use of ultrasound to increase the effectiveness of isokinetic exercise for the knee osteoarthritis.

[23]. Gail D et al (2000) determined the effectiveness of manual physical therapy and exercise in osteoarthritis of knee by randomised control trail.

[24]. Susan B Osullivan EdD.PT(2001) Physical Rehabilitation Assessment and treatment Fourth edition Published by Jiteneder p vij Jaypea Brothers medical publisher (p) Ltd.EMCA House New Deli India.

[25]. Robertson VJ Ward AR Dangers in extrapolating in vitro uses of therapeutic ultrasound.phys Ther 1996,76(1);78-79

[26]. Bakers KG Robertson VJ,Duck FA:A review of therapeutic ultrasound:biophysical effects.Phys Ther 2001,81(7):1351-1358.

[27]. Vas Burton AK, Waddell G Tillotson KM et al information advise to the patiets with back pain may have positive effect.A randomized control trial of the vovel educational booklet in primary care spine $1999 ; 24: 2,484-91$

[28]. Delorme T,Watkins A.Techniques of progression exercise.Arch phys Med Rehabil 1948:14:377-81 kisner C Colby LA. Therapeutic Exercise: foundation and Techniques.Philadelphia: FA Davis Company 1990:109:109-46 